Archives

$45 \mid 2010$

La preuve en histoire

\title{
La preuve par le carnet B
}

Jean-Pierre Deschodt

\section{(2) OpenEdition}

Journals

Édition électronique

URL : http://journals.openedition.org/ccrh/3567

DOI : $10.4000 /$ ccrh.3567

ISSN : $1760-7906$

Éditeur

Centre de recherches historiques - EHESS

\section{Édition imprimée}

Date de publication : 25 avril 2010

Pagination : 181-193

ISSN : 0990-9141

Référence électronique

Jean-Pierre Deschodt, "La preuve par le carnet B », Les Cahiers du Centre de Recherches Historiques [En ligne], 45 | 2010, mis en ligne le 27 février 2012, consulté le 10 décembre 2020. URL : http:// journals.openedition.org/ccrh/3567 ; DOI : https://doi.org/10.4000/ccrh.3567

Ce document a été généré automatiquement le 10 décembre 2020.

Article L.111-1 du Code de la propriété intellectuelle. 


\title{
La preuve par le carnet B
}

\author{
Jean-Pierre Deschodt
}

1 Quel est le statut du fait dans l'histoire ? Et dans quelle mesure peut-il être avancé à titre de preuve?

2 Pour le comprendre, il peut être utile de comparer le fait historique au fait scientifique (celui des sciences « dures », non « humaines »). Ce serait une naïveté de penser que, pour la science, le fait est immédiatement donné (à l'observation). En réalité, pour établir un fait, il faut commencer par discriminer, au sein d'un réel chatoyant, complexe et muable, des régularités et des consécutions certaines, en écartant quelques relations plus apparentes qu'effectives, établissement qui constitue une œuvre de la raison scientifique investiguant ce réel et le soumettant, comme disait Lord Bacon, «à la torture ». Mais ce fait obtenu n'explique rien à lui seul. Pour en rendre compte, l'esprit conçoit des hypothèses (et, de façon plus large, des théories) explicatives. Ces dernières étant élaborées, le scientifique en déduit les conséquences : si mon hypothèse (ou ma théorie) est vraie, tel fait doit pouvoir être observé. C'est ainsi que Einstein a fait dépendre toute sa théorie de la relativité généralisée d'une seule observation. C'est au terme de cette méthode, que Claude Bernard a qualifiée d'«hypothético-déductive », que le fait peut « faire » preuve.

Qu'en est-il maintenant de l'histoire ? Les faits y sont-ils donnés, construits, prouvent-ils quelque chose?

4 La tâche principale à laquelle l'historien se voit confronté est la détermination du fait. Mais ce dernier étant comme invisible, puisque passé et définitivement perdu (et ne pouvant faire l'objet d'une expérience réitérable, comme il en va dans les sciences de la nature), il est au départ inconnu et, en quelque sorte, problématique aux yeux du chercheur, qui sera naturellement amené à mobiliser tout son talent pour s'interroger sur la nature des événements qui font l'histoire. Pour parvenir au fait, l'historien s'emploiera à reconstituer une tranche du passé par la vertu d'une lecture critique des documents, lesquels documents constituent un cadre naturel à la connaissance en général, et aux causes en particulier. Il s'agira pour lui de maitriser l'administration de l'élément documentaire mettant en valeur le principe de concaténation à travers un questionnement précis qui réponde à des critères méthodologiques ayant fait leur 
preuve. Ai-je le texte exact et intégral tel qu'il a été écrit par l'auteur? (critique d'intégrité). Ce texte est-il bien de l'auteur auquel on l'attribue? (critique d'authenticité). Qu'est-ce que l'auteur a voulu dire ? A-t-il cru ce qu'il a dit? (critique de sincérité) Etait-il fondé à croire ce qu'il a cru? (critique d'autorité). Toutes ces interrogations correspondent à des éclairages différents : d'ordre psychologique, littéraire, narratif, et confèrent au récit historique toute sa qualité et toute sa cohérence.

Une difficulté mérite cependant d'être soulignée: une même série de faits peut déboucher sur des livres d'histoire différents selon le but que le chercheur se sera donné. C'est que les faits historiques peuvent être considérés sous trois angles différents : dans ce qu'ils ont de collectif et de durable ; dans ce qu'ils présentent d'individuel et de passager; dans ce qui relève de l'« invisible ${ }^{1}$ enfin.

Si l'on veut reconstituer l'histoire de la surveillance administrative des citoyens d'un pays à une époque donnée - telle est notre intention dans le travail qui va suivre - il semble judicieux de commencer par s'intéresser aux motifs de la suspicion étant entendu que le statut de suspect ne peut se comprendre qu'à travers l'étude nominative des cas dûment recensés dans le fichier.

7 Mystérieux carnet B aux formes énigmatiques, qui se présente sous l'aspect d'un registre sur lequel sont rassemblés des individus français ou étrangers. Carnet B aux fonctions inconnues qui a mis longtemps à révéler son existence et sa véritable nature. Carnet aux secrets bien gardés qui au nom de la défense du territoire ou plutôt de celle de l'État radical est resté enfoui dans les sous-sols ministériels. À partir de ses premiers éléments, nous comprenons mieux pourquoi les chercheurs ont vu leurs investigations buter sur un front du secret. La réponse qu'a reçue, le 15 novembre $1965^{2}$, Jean Jacques Becker du directeur général de la Sûreté nationale, à l'enquête qu'il menait sur les archives du carnet $\mathrm{B}$, illustre bien ce problème. En affirmant : « aucune documentation n'existe plus à l'échelon national sur ce carnet. Les archives du fichier central de la Sûreté nationale ont été détruites en 1940, lors du repli des services ", Maurice Grimaud formait une réponse qui parut être la plus plausible et la plus circonstanciée. Or à la suite d'un accord intervenu en $1992^{3}$ entre les gouvernements français et russe organisant la restitution des archives de la Sûreté nationale, il est apparu que la thèse de la destruction des sources n'était plus à l'ordre du jour et que l'on pouvait supposer que les nazis puis les soviétiques avaient eu le temps de compléter leurs fiches de renseignement à l'aide des dossiers du ministère de l'Intérieur.

8 Quiconque s'intéresse à l'organisation du contre-espionnage français entre les deux guerres détient avec le fonctionnement du carnet $\mathrm{B}$, une clef qui permet l'élargissement du champ d'investigation dans un cadre où les informations répertoriées s'articulent autour de règles précises. L'instruction secrète du $1^{\text {er }}$ novembre $1912{ }^{4}$ en définit le mode opératoire : il s'agit d'instruire un fichier où seront inscrites les personnes dangereuses pour l'ordre intérieur en cas de troubles, de conflit ou de tension politique. Révisée le 10 février $1922^{5}$, à l'initiative du ministre de l'Intérieur, Maurice Maunoury, le carnet B semble alors inadapté au nouveau danger qui menace le pays, il ne comporte pas «les noms de certains individus notoirement acquis aux idées extrémistes ». Force est de constater que la vie au sein du carnet B s'organise autour de plusieurs acteurs. Le premier est un administrateur, c'est-à-dire celui qui décide de l'inscription, le second est le rédacteur, celui qui compose le rapport et le tout est un suspect. 


\section{Suspect}

9 De quelle manière devient-on suspect ? Sur quel critère est-on choisi pour figurer dans la catégorie des indésirables ? Sont considérés comme suspects les Français et étrangers qui divulguent aux puissances ennemies des renseignements concernant la Défense nationale et qui sont donc capables de se «livrer à l'espionnage et de prêter leur concours à des manœuvres préjudiciables » à l'indépendance nationale. Mais aussi ceux qui peuvent perturber la mobilisation, détruire une œuvre stratégique et attaquer des magasins d'approvisionnement. C'est pourquoi le fichier se subdivise en trois groupes. Les étrangers et les Français suspects d'espionnage constituent les deux premiers; les Français qui représentent « réellement » un danger pour l'ordre intérieur, le dernier. Or, au sein du premier groupe, des étrangers non suspects peuvent en fonction de leur situation géographique, se trouver mêlés au carnet B :

- ceux qui habitent une maison qui se trouve au-dessus ou en dessous d'un ouvrage d'art d'intérêt stratégique ;

- ceux qui habitent une maison isolée située : d'une part à moins d'un kilomètre d'un ouvrage d'art ou d'une voie ferrée stratégique; d'autre part, à plus d'un kilomètre d'une ville, d'un village, d'un hameau, d'un groupe de maisons ;

- ceux qui habitent une propriété close, qui se trouve en bordure d'une voie ferrée stratégique, ou qui est attenante à un ouvrage d'art d'intérêt stratégique ${ }^{6}$.

Les points sensibles qui sont à protéger en première urgence dans les différents secteurs impliquent une connaissance approfondie des intentions des habitants de nationalité étrangère qui pourraient se révéler être des facteurs favorables à l'espionnage ou au sabotage. Ici c'est un étranger suisse, Emile Gervaix ${ }^{7}$, directeur d'une laiterie dont le domicile est proche des ouvrages fortifiés; il possède un véhicule avec lequel il fait de nombreux déplacements dans son pays d'origine, son nom est inscrit au carnet B le 25 mai 1937 d'autant que son comportement est peu apprécié par la population locale. Là c'est une famille belge Lacroix ${ }^{8}$ dont les membres sont hypocrites, dénués de scrupules et vivant d'expédients, habite à environ 300 mètres d'une route stratégique. On trouve aussi un Italien ${ }^{9}$ habitant à moins d'un kilomètre d'un ouvrage militaire savoyard. Ces quelques exemples ne constituent pas au demeurant une avalanche d'inscriptions.

11 En prenant l'exemple du plan de protection des deux cantons de Morteau où une vingtaine de lieux sont répertoriés, nous allons pouvoir étendre notre investigation à tout un secteur de défense frontalière. La description de la station électrique fait état de la présence d'un sujet Suisse, non suspect, dans un rayon de 1 kilomètre. À un autre endroit, au pont de la Roche, on note la présence de quatre étrangers non suspects, puis au pont de Morteau, un homme de nationalité italienne y réside à 30 mètres. Ces étrangers sont signalés, et se sont certainement retrouvés inscrits au carnet B. À côté de cet espionnage de situation, les Français ont aussi leur place dans le système de lutte contre les menées étrangères. L'inscription au $2^{e}$ groupe procède d'un certain nombre de facteurs mis en valeur par une enquête minutieuse. Tout d'abord on évalue son métier, ses déplacements, ce qui sous-entend de quel moyen de locomotion il dispose. Se pose ensuite la fiabilité de ses relations, de ses amitiés. Se trouve-t-il aux sommiers judiciaires ? De quelle nature ont été ses condamnations? Est-il naturalisé? A-t-il été sujet alsacien, lorrain? Afin de compléter ses investigations, il nous semble opportun de nous arrêter sur la famille Offenstadt ${ }^{10}$. D'origine bavaroise, les Offenstadt ont été naturalisés en 1890 . Très vite, ils 
se sont lancés dans les maisons d'édition licencieuses et dans la diffusion de publications obscènes, notamment «La Culotte rouge ». Au regard du carnet $\mathrm{B}$, leurs activités ont été présentées comme anti-militaristes parce qu'elles déconsidéraient l'armée française à l'étranger.

Il s'agit maintenant de s'intéresser au troisième groupe, les suspects français de l'ordre intérieur. Ces fameux extrémistes dont nous avons parlé tout à l'heure. Par idées extrémistes, il faut entendre tout individu qui est susceptible d'entretenir des troubles violents au moyen d'une propagande antipatriotique et libertaire qui favorise «l'action directe ", c'est-à-dire rébellion, appel à l'émeute et au meurtre. Aussi par la lecture d'une centaine de dossiers, nous allons essayer de déterminer par ordre d'importance ces fameuses catégories extrémistes en nous appuyant sur les motifs de leur inscription :

- les anarchistes et les syndicalistes : les amis de Sébastien Faure, des individus violents et exaltés pouvant occuper les fonctions de secrétaire de la Bourse du travail. Propagandiste excité faisant des provocations contre les militaires, favorables à l'insoumission et aux grèves insurrectionnelles ;

- les communistes: Organisateurs de manifestations communistes, meneurs de grèves, apôtres de la guerre civile, secrétaires du rayon communiste, présidents d'un syndicat municipal communiste, apologie de faits qualifiés de crime et membres du comité central;

- les autonomistes: francophobie, autonomisme breton, agitateur autonomiste manifestant des sentiments germanophiles;

- les fascistes : membres du fascio ou du front du travail allemand.

13 Il faut avoir à l'esprit ce que signifiait une inscription au carnet $\mathrm{B}$ : l'individu pouvait être mis en état d'arrestation sur une simple décision du préfet lequel devait juste remplir un formulaire blanc qui était joint au dossier. Ces mandats d'arrestation étaient donc préparés à l'avance et annexés à chaque dossier au nom de l'article 10 du code d'instruction criminelle. C'est sous cette forme légale qu'un préfet pouvait exercer les attributions de police judiciaire d'un juge d'instruction. Il est à noter que parfois le carnet $\mathrm{B}$ réservait des surprises désagréables à ses membres. Un officier de l'armée active, Chevalier de la Légion d'honneur, s'est vu opposer un refus de ses supérieurs au mariage avec la fille de Moïse Offenstadt inscrit au carnet $B$ dans le groupe $n^{\circ} 2$.

\section{Administrateur}

Ordonnées par les préfets et les généraux commandant les corps d'armée ${ }^{11}$ qui doivent suivre " personnellement " l'examen des dossiers, les inscriptions au carnet B sont soumises en cas de différend entre les deux parties civile et militaire, à la responsabilité commune des ministres de la Guerre et de l'Intérieur. Aussi l'individu suspect se retrouve en fiche dans chaque brigade de gendarmerie et dans chaque préfecture dont dépend son domicile ainsi que dans chaque état-major de Corps d'Armée dont dépend son affectation ${ }^{12}$. Ces folios mobiles, transmis entre les différents centres de gendarmeries doivent permettre de suivre ses déplacements sans que l'intéressé en soit informé, permettant ainsi de constituer les petits secrets d'État, utiles à la neutralisation des indésirables en cas de conflit, de tension et de mobilisation. 


\section{La radiation}

15 Sur proposition des autorités administratives notamment du préfet, et après acceptation du ministre de l'Intérieur, le carnet B peut faire l'objet de révisions en ce qui concerne les individus qui se sont amendés ou n'étant plus susceptibles de fomenter ni d'exercer une action révolutionnaire à titre individuel ou collectif. En outre, cette révision est rendue nécessaire pour rayer les inscrits qui sont décédés, disparus ou partis dans d'autres pays depuis plus de deux ans. C'est dans cet esprit que le ministre de l'Intérieur, Maunoury, propose, dès 1922, qu'annuellement le carnet soit purgé de ces éléments; il faut néanmoins attendre $1926{ }^{13}$ pour qu'une nouvelle révision des listes soit effectuée à la suite d'enquêtes. En pratique comment faisait-on pour s'amender? Pour raison médicale, un suspect atteint par une défaillance physique ou victime d'une pathologie évolutive ne doit plus être considéré comme une menace. Le cas de Ferdinand Fiacre ${ }^{14}$, suspect français $2^{\mathrm{e}}$ groupe, courtier en cartes postales est un exemple frappant. Ce « sujet alsacien lorrain " parcourt toute la région à vélo et vend ses photographies en Allemagne ; frappé de congestion et de paralysie en mai 1926, le préfet de police demande neuf mois plus tard sa radiation.

Pour raison politique: des individus qui ne manifestent plus la même passion pour les idées révolutionnaires parce qu'ils se sont retirés de la vie politique ou syndicale, se sont donc amendés par désintérêt pour le militantisme. C'est le cas du dénommé Victor Delagarde ${ }^{15}$ qui n'est plus considéré comme antimilitariste après avoir diffusé et édité des documents contre le comité central du parti communiste aboutissant à son exclusion du rayon en 1924. André Fonteny, s'est lui aussi assagi, inscrit en 1922, au carnet B de la Seine dans le $3^{e}$ groupe, pour les motifs suivants :

en 1919, il a adhéré à la 5e section de la S.F.I.O., et pendant un certain temps il a

même assisté aux réunions de ce groupement en tenue de capitaine.

Présenté comme un porteur du drapeau rouge à l'occasion des manifestations, il rompt avec ses anciennes activités en acceptant les fonctions de secrétaire du député radicalsocialiste de la Drôme, André Escoffier, Fonteny est donc radié en 1926 du carnet B. Peuton se retrouver inscrit deux fois au carnet B ? En théorie rien dans les instructions ne s'y oppose cependant le cas est assez rare. Rayé une première fois sous le motif « disparu depuis plus de deux ans", Louis Gorion ${ }^{16}$ fut retrouvé et réinscrit aussitôt mais sa présence n'étant plus constatée dans les milieux extrémistes de la région parisienne, il fut quelque temps plus tard définitivement retiré du carnet $B$.

\section{Rédacteur}

En lien avec le préfet, mais surtout avec le contrôleur général des services de surveillance du territoire, le Commissaire spécial a comme attribution la défense de la sûreté de l'État et c'est en cela qu'il est le collecteur total des informations sur le contre espionnage. À la tête d'un secteur, il se doit de mener des enquêtes sur des personnes civiles ou militaires pour surveiller la propagande extrémiste et les menées anticonstitutionnelles, syndicalistes ou anarchistes et de s'opposer ainsi aux tentatives de manipulations, il représente le fond territorial d'information. Sa participation au carnet B est des plus importantes puisqu'il participe aux rapports qui composent la substance du dossier classé B. La multiplication des enquêtes laisse à penser que les effectifs de la police ne suffisent 
plus à faire face à la demande. À ce niveau de la discussion, nous devons nous poser la question sur la nature des informateurs. Les mouchards, rétribués sur des fonds spéciaux certainement, sont-ils en nombre suffisant pour récolter toutes les informations demandées? On peut à ce stade avancer l'idée que la police ait pu bénéficier de la délation. Or s'il fallait attendre qu'un délateur se manifeste pour pouvoir agir, les enquêtes auraient été particulièrement longues et fastidieuses. Que fallait-il faire? Discrètement, on a alors étendu le rôle de surveillance aux auxiliaires de l'administration. Selon les termes de l'instruction interministérielle Guerre/Intérieur du 16 juin 1926 :

les renseignements de contre-espionnage recueillis par les agents auxiliaires dénommés ci-après: gardes champêtres, cantonniers, facteurs ruraux, gardes forestiers, éclusiers, gardes de la navigation fluviale seront centralisés par la brigade de gendarmerie sur la circonscription de laquelle ils sont en fonction et transmis au commissaire spécial, chef de secteur pour information. ${ }^{17}$

Dans une note du 21 juillet $1933^{18}$, sur la participation de la gendarmerie au service de contre-espionnage, estampillée très secret, il est précisé que le concours de ces agents auxiliaires allait permettre à la gendarmerie « d'exercer, sur l'ensemble du territoire, une surveillance très serrée et particulièrement intéressante ». Cette participation active à la surveillance est encore plus ancienne puisque dès 1919, les commissaires spéciaux ont été mis en possession des listes de gardes-champêtres, agents de police, gardes forestiers, agents du service de la navigation et poste de douane.

Et seize années plus tard lorsque le commissaire spécial de la circonscription régionale de Dijon envoie son plan de protection du secteur de Morteau au contrôleur général, il n'oublie pas d'intégrer les auxiliaires qu'il a recrutés entre autres quelques 130 cantonniers et 45 facteurs ruraux. Voici les dispositions générales qui ont été définies dans l'instruction interministérielle du 26 novembre 1924 (article 41) ${ }^{19}$ :

Les agents auxiliaires doivent surveiller et signaler au chef de secteur :

- Les individus qui s'intéressent aux tracés des routes nouvelles.

- Les individus aux allures suspectes qui observent les manœuvres militaires [...].

- Les automobilistes ou cyclistes dont les allures paraissent suspectes [...].

- Les individus qui font de fréquents voyages à l'étranger sans y être appelés par leur profession [...].

- Les musiciens ambulants, colporteurs ou autres individus exerçant des professions nomades.

- Les déserteurs étrangers ou français [...].

- Et, d'une façon générale, toute menée suspecte et tout fait pouvant intéresser la sûreté de l'État.

\section{L'engrenage des années trente}

Quant aux étrangers ayant commis des faits réputés dangereux pour l'État, ils n'entrent dans aucune rubrique spécifique de cette classification. Leurs dossiers doivent en effet être adressés au ministre de l'Intérieur afin qu'il puisse examiner "sans retard des propositions d'expulsion ${ }^{20}$. En évoquant le cas des étrangers, il semble nécessaire d'introduire une distinction, qui a été matérialisée dans le cadre général de classement entre les suspects français et ceux d'une autre nationalité. Les grands ensembles n'empêchent pas la multiplication des sous-dossiers, rendue d'autant plus nécessaire que le classement des folios mobiles concernant les étrangers reprend l'ordre alphabétique de chaque pays et diffère de celui des Français dont le mode de sélection reste attaché au nom patronymique. Bientôt, on établit pour chaque pays, un état numérique. 
L'Allemagne ${ }^{21}$ devient la nationalité numéro un, se distinguant de l'Autriche et de la Hongrie qui portent respectivement les numéros deux et trois ; on attribue à l'Espagne le chiffre huit et dix-neuf pour la Russie. Ainsi, enregistrés sous la nationalité numéro six ${ }^{22}$, les «indésirables» italiens font l'objet depuis le mois d'août 1930 et dans un certain nombre de départements frontières, d'un « classement spécial » que la circulaire du 4 janvier $1932^{23} \mathrm{du}$ ministre de l'Intérieur impose à l'ensemble du territoire national. Ce carnet particulier se compose d'étrangers de nationalité italienne suspects au point de vue national et qui, «tout en évitant de se livrer en temps normal à des agissements suspects, néanmoins susceptibles de le devenir soit à l'occasion de troubles sociaux, soit en cas de tension politique ", sont à même de commettre des actes préjudiciables à la Défense nationale. Le mode de fonctionnement et d'attribution du nouveau carnet est identique au précédent. Quels sont les types d'Italiens visés par cette circulaire ? Une note de la Préfecture de Police du 20 janvier 1932 nous renseigne à ce sujet: «Un certain nombre de fascistes qui jouent le rôle de provocateurs, sans que les faits aient pu être nettement établis, y seront inscrits ».

Le caractère indéterminé de la définition donne au carnet spécial une forme d'arbitraire à travers laquelle les services compétents ont toute latitude à transformer le doute en certitude. Ce système est dirigé contre la seule nationalité numéro six (l'Italie), jusqu'à l'intervention du ministre de la Guerre, Edouard Daladier qui, au début de l'année 1933, demande à son collègue de l'Intérieur, Camille Chautemps, d'étendre à tous les indésirables étrangers, l'exception italienne. Le 25 janvier $1933^{24}$, le directeur de la Sûreté générale communique aux préfets l'extension du dispositif. Sont désormais concernés tous les étrangers de toutes nationalités dont les agissements ne peuvent pas être soumis aux procédures d'éloignement, mais qui paraissent néanmoins sujets à se transformer en agitateurs dangereux pour l'ordre intérieur. Toutefois, à la demande expresse des ministères de la Guerre et de l'Intérieur, le «caractère rigoureusement secret » de ce carnet est impératif : à aucun moment les inscrits ne doivent «se douter de la mesure dont ils sont l'objet ». Prévention qui répond autant à un souci de discrétion et à une volonté d'efficacité qu'à une raison diplomatique, les personnages concernés étant en relation constante avec les ambassades étrangères; c'est pourquoi les commandants des brigades de gendarmerie ont reçu des consignes strictes de ne pas diligenter des interrogatoires qui auraient comme résultat d'éveiller les soupçons et d'embarrasser le gouvernement. En cas de tension politique grave, le sort des étrangers est alors régenté par l'instruction du ministre de la Guerre du 19 juillet 1931, qui précise le cadre dans lequel les prescriptions concernant le carnet «spécial» s'appliqueront. Les mesures d'exception que prévoit l'instruction confèrent à ce carnet des moyens préventifs et répressifs. Les suspects mobilisables des puissances ennemies doivent être appréhendés au premier jour de la mobilisation et seront consignés à la disposition de l'autorité militaire pour les envoyer soit dans les camps de concentration, soit hors des frontières nationales. En revanche, les ressortissants des nations amies verront simplement leur surveillance s'accentuer.

\section{Le carnet B du département de la Seine}

23 Afin de mieux saisir la valeur qualitative et quantitative de ce carnet, nos recherches se sont tournées vers le fonds de la Préfecture de Police, archives que Jean-Jacques Becker n'a pas consultées et qui ne faisaient pas parties des 86 demandes de renseignement 
adressées aux directeurs départementaux ${ }^{25}$. La présence de plusieurs cartons traduit un certain potentiel du carnet B dans le département de la Seine. En mars $1933{ }^{26}$, les trois groupes recensés à la préfecture de police comptent un nombre d'inscrits particulièrement faible et, même si deux ans plus tard une augmentation est enregistrée, il reste encore dérisoire par rapport à une population parisienne estimée à 2,7 millions d'habitants. D'ailleurs le commandant Mermet, officier de liaison du gouver-nement militaire de Paris, au nom du général Gouraud, s'interroge en mars $1935{ }^{27}$, sur la capacité du département de la Seine à recenser les candidats espions. Il parait fortement improbable que seuls 26 espions officient dans cette circonscription, ce qui conduit les Renseignements Généraux à revoir certains cas individuels et à concentrer les informations afin que le préfet puisse intégrer de nouveaux noms dans les deux premiers groupes.

\begin{tabular}{|l|l|l|}
\hline & 1933 & 1935 \\
\hline Effectif du $1^{\text {er }}$ groupe & 1 & 13 \\
\hline Effectif du $2^{\text {e }}$ groupe & 6 & 13 \\
\hline Effectif du $3^{\mathrm{e}}$ groupe & 266 & 311 \\
\hline
\end{tabular}

Qu'advient-il dans le même temps du carnet $B$ spécial, répertoire des indésirables de toutes nationalités? Les renseignements collectés par le service des Affaires de sûreté générale de la Préfecture de Police ${ }^{28}$ permettent l'établissement d'une liste de 146 étrangers, ce qui est, là encore, peu par rapport à la liste de 5.635 personnes que la direction des Renseignements Généraux a transmise dès le 11 octobre $1932{ }^{29}$ au ministère de l'Intérieur.

Le Carnet B spécial de la Préfecture de Police

\begin{tabular}{|l|l|}
\hline & 1935 \\
\hline Allemands & 3 \\
\hline Italiens & 121 \\
\hline Espagnols & 4 \\
\hline Russes soviétiques & 3 \\
\hline Russes blancs & 2 \\
\hline Polonais & 2 \\
\hline
\end{tabular}

Individus suspects au R.G. de la Préfecture de Police 


\begin{tabular}{|l|l|}
\hline Allemands & 51 \\
\hline Italiens & 1733 \\
\hline Espagnols & 574 \\
\hline Russes soviétiques & 669 \\
\hline Russes blancs & 1009 \\
\hline Polonais & 397 \\
\hline
\end{tabular}

Ces éléments statistiques mettent en évidence, ainsi que le montre alors la note administrative de la préfecture de police, que le carnet B « spécial » est «notoirement insuffisant », et qu'il faut procéder à l'intégration des éléments contenus dans le relevé des R.G. Pour ce faire, le $1^{\text {er }}$ bureau réclame que soient transmises les listes complémentaires «avec les renseignements, par rapport séparé concernant chacun de ces étrangers». Nous ne connaissons pas le résultat de ces investigations, les archives restent muettes à ce propos. En guise de comparaison et selon les chiffres connus ${ }^{30}-$ l'état descriptif du carnet $\mathrm{B}$ à la veille de la Première Guerre mondiale n'ayant pu être retrouvé - si notre attention se porte sur les 2.500 suspects identifiés dans toute la France, nous constatons que le nombre des inscrits du département de la Seine représente en 1935, $20 \%$ de l'effectif global de 1914 ; ce qui incline à envisager une augmentation de l'activité de ce même carnet. Maintenant, si nous nous référons à l'échantillon des 5.635 indésirables étrangers comptabilisés par les Renseignements Généraux sis 7, Boulevard du Palais, la différence en terme de population est deux fois plus importante que celle du carnet B en 1914, dont les éléments chiffrés couvrent le territoire national et non pas simplement le ressort de la Préfecture de Police. Bien sûr, il ne s'agit que de candidats à l'inscription, mais le simple énoncé de leur nombre indique l'existence d'une réserve qui entretient la vitalité du système.

\section{Un nouveau cadre juridique}

Au 30 décembre $1932^{31}$, la Chambre des députés adopte une loi qui définit les contenus de la liberté individuelle, supprime l'article 10 et retire donc aux préfets la légalité d'une arrestation immédiate des individus suspects. Le carnet dans son principe est très sérieusement menacé pour sa partie française. Il faut attendre la loi du 25 mars $1935^{32}$ et la rédaction d'un nouvel article 10, pour que les préfets des départements et le préfet de Police à Paris puissent,

s'il y a urgence, faire personnellement ou requérir les officiers de police judiciaire, chacun en ce qui les concerne, de faire tous actes nécessaires à l'effet de constater les crimes et délits contre la Sûreté intérieure ou la sûreté extérieure de l'État [...].

L'arrestation ne s'étend pas au nom de tous les crimes et délits mais seulement lorsque la sécurité de l'État tant dans son territoire qu'en dehors de celui-ci, est en danger. Dès que le préfet fait usage de ses pouvoirs judiciaires, il est tenu dans un délai de 24 à 72 heures d'en aviser le procureur de la République et de transmettre les pièces à ce magistrat qui se saisit de l'affaire. Le système juridique qui accompagne le carnet $B$ est ainsi sauvegardé. 
Ae la nécessité d'harmoniser les centres d'informations, les responsables de la Sûreté nationale envisagent une simplification du système en améliorant la technique de classement. La circulaire du 16 juillet $1938^{33}$, cosignée par les ministères de la Guerre et de l'Intérieur, entraîne une clarification structurelle par la création d'un $4^{e}$ groupe qui dorénavant se substi-tuera au précédent carnet spécial. Tous les étrangers dangereux pour l'ordre intérieur qui «par leurs actes, discours, écrits, propagandes, seront considérés comme dangereux ou ceux qui seront estimés susceptibles de se transformer en agitateurs à la faveur d'un incident sérieux » sont censés rejoindre ce $4^{\mathrm{e}}$ groupe. La réunion des deux carnets $\mathrm{B}$, ordinaire et spécial, en un instrument unique désormais divisé en quatre groupes constitue un fait déterminant dans le contrôle prudent de l'élément suspect étranger et français. laissent à l'autorité administrative, en l'occurrence le préfet et le général commandant un corps d'armée, une grande latitude dans l'admission ou le refus d'un individu au carnet B. Les motifs de l'inscription peuvent tenir, on l'a vu, à un lieu d'habitation géographiquement sensible mais, bien plus simplement, d'activités politiques et syndicales qui favorisent tacitement ou non les intérêts d'une nation étrangère.

Mais le carnet $\mathrm{B}$ est loin de répondre à l'efficacité à laquelle son créateur avait pensé. D'une part, il apparait que le but n'est pas clairement défini si ce n'est le désir de neutraliser le suspect lequel reste un terme protéiforme au contenu assez vague. Autant les deux premiers groupes du carnet répondent à des critères précis soulignés par la loi de 1934 sur l'espionnage, autant les deux derniers groupes ont une ligne administrative floue, insaisissable, sujette à interprétation, à contestation, sans véritable orientation générale autre que celle de la défense de l'ordre intérieur. D'autre part, il n'existe pas de frontières clairement établies entre le carnet $B$ et les listes d'indésirables constituées par les services de la police administrative, comme celles des propagandistes révolutionnaires (P.R.), des anarchistes de la Préfecture de Police dont les domiciles sont vérifiés tous les 15 jours (le cas de Marcel Hasfeld) ${ }^{34}$ et les individus classés Z (Gustave Bouvet) ${ }^{35}$.

La dimension politique du carnet est primordiale et toute la difficulté réside dans sa capacité à traiter pareillement les questions d'espionnage et d'activisme. Efficace dans un secteur plutôt que dans l'autre, le ministère de l'Intérieur privilégie numériquement dans le cadre du carnet, la lutte contre les éléments étrangers capables d'action violente et non plus, comme c'était le cas avant 1914, celle tournée contre les Français anarchistes qui représentaient alors $60 \%$ de l'effectif total. On en a justifié en droit toutes les applications pratiques en vertu d'un principe d'exception qu'aucun gouvernement ${ }^{36}$ ne voudra ni modifier ni supprimer; il faut attendre le 18 juillet $1947^{37}$ pour que les ministres Paul Coste-Floret et Edouard Depreux abrogent le fameux carnet.

\section{NOTES}

1. Philippe Ariès, Essais de mémoire, Paris, Seuil, 1993, p. 40.

2. Jean-Jacques Becker, Le carnet B, Paris, Klincksieck, 1973, p. 123 (note 1). 
3. Bernard Devaux, «Les Archives de la Sûreté rapatriées de Russie », Gazette des archives, 1997, p. 78-85.

4. Instruction du $1^{\mathrm{er}}$ novembre 1912, mise à jour le 12 décembre 1922, Archives Nationales (AN), CAC, vers. 19940 500/1328.

5. Archives de la Préfecture de Police (APP), BA/2273, Circulaire du ministre de l'Intérieur du 10 février 1922.

6. APP, BA/2273, Circulaire du ministère de l'Intérieur, 15 février 1922.

7. AN, CAC, vers. 19940 500/1542.

8. Inscrit au carnet B de la Meuse le 15 novembre 1938.

9. Louis Morras inscrit au carnet B le 18 septembre 1939.

10. AN, CAC, vers. 19940466 art 15 dossier 1191 .

11. Instruction du $1^{\mathrm{er}}$ novembre 1912, mise à jour le 12 décembre 1922.

12. Ibid.

13. APP, BA/2273, Note sur la révision du carnet $B$.

14. AN, CAC, vers. 19940 445/112, dossier 10490.

15. AN, CAC, vers. 19940 440/137 dossier 11501.

16. AN, CAC, vers. 19940 448/329 dossier 27658.

17. AN, CAC, vers. 19940 500/1328.

18. Ibid.

19. AN, CAC, vers. 19940 500/1328, Note sur la participation de la gendarmerie au service du contre-espionnage, p. 20-21.

20. Instruction du $1^{\mathrm{er}}$ novembre 1912 , op. cit.

21. APP, BA/2273, Note du préfet de Police, 17 octobre 1933.

22. APP, BA/2273, Note du préfet de police, 20 janvier 1932.

23. Ibid.

24. APP, BA/2273, Circulaire du ministère de l'intérieur, 25 janvier 1933.

25. Jean-Jacques Becker, op. cit., p. 208.

26. APP, BA/2273, Note au sujet de l'application du carnet B dans le département de la Seine, mars 1933.

27. APP, BA/2273, Note de la préfecture de police, mars 1935.

28. Ibid.

29. Ibid.

30. Cité, notamment par Jean-Jacques Becker, op. cit., p. 128.

31. APP, BA/2273, Circulaire du ministre de l'Intérieur, 25 janvier 1933.

32. APP, BA/2273, Note pour le directeur des renseignements généraux, 2 avril 1935.

33. APP, BA/1774, Rectificatif à l'instruction du $1^{\mathrm{er}}$ novembre 1912, 16 juillet 1938.

34. AN, CAC, vers. 19940 451/67, dossier 5943.

35. AN, CAC, vers. 19940 434/547, dossier 45454.

36. Le gouvernement du Front populaire a utilisé le carnet $\mathrm{B}$ à l'encontre de grévistes. Maria Iverlend a été inscrite dans celui de la Somme, le $1^{\mathrm{er}}$ octobre 1936 avec comme motif : « extrémiste militante depuis de longues années. A pris une part très active dans la conduite du mouvement de grèves et occupation des usines juin-août 1936. Violente et exaltée ». AN, CAC, vers. 19940 500/4611.

37. APP, BA/1774, Suppression de l'instruction ministérielle sur le carnet B. 


\section{AUTEUR}

JEAN-PIERRE DESCHODT

ICES, La Roche-sur-Yon 